\title{
Incorporação de Lodo Industrial em Compósitos de Resina Poliéster
}

\author{
Ivonete 0. Barcellos, Antônio C. de Souza, Anna E. Selke \\ Departamento de Química, Universidade Regional de Blumenau
}

\begin{abstract}
Resumo: A incorporação de lodo industrial (L) e fibra de crisotila (FC) na preparação de compósitos com resina poliéster (P) é uma possível alternativa para minimizar o acúmulo de resíduos sólidos. A caracterização dos compósitos estruturais com estes reforços indicou diminuição nos valores de densidade quando comparados com compósitos reforçados com fibra de vidro. Os valores máximo e mínimo de resistência ao impacto Izod, para formulações do tipo P/L/FC, foram 29,1 e 12,6 J.m . A incorporação de lodo contribuiu para o aumento da capacidade de isolamento térmico de compósitos com fibra de crisotila, além de preservar a estabilidade térmica da matriz polimérica. Os resultados indicaram que apesar de provocar perdas mecânicas, a incorporação de lodo em compósitos com matriz de poliéster resulta em laminados com densidade reduzida e baixa condutividade térmica.
\end{abstract}

Palavras-chave: Compósitos, lodo industrial, propriedade térmica.

\section{Incorporation of Industrial Sludge in Polyester Resin Composites}

Abstract: The incorporation of industrial sludge (L) and chrysotile fiber (FC) in the preparation of composites with polyester resin $(\mathrm{P})$ is a possible alternative to minimize the accumulation of solid wastes. The characterization of the structural composites with these reinforcements indicated reduction in density values when compared with glass fiber reinforced composites The maximum and minimum values of Izod impact resistance for P/L/FC formulations were 29.1 and 12.6 J.m ${ }^{-1}$. The incorporation of sludge led to an increased thermal isolation capability for composites with crysotile fiber, also preserving the thermal stability of the polymeric matrix. The results indicated that although there are damages in mechanical properties, the incorporation of sludge in composites with polyester matrix results in mils with reduced density and low thermal conductivity.

Keywords: Composites, industrial sludge, thermal properties.

\section{Introdução}

O crescimento populacional e o aumento da atividade industrial acentuam consideravelmente a produção de resíduos, contribuindo negativamente para o impacto ambiental. Os efeitos do acúmulo de resíduos podem ser percebidos nas alterações da qualidade do solo, do ar e da água ${ }^{[1]}$. A recuperação de águas naturais é o maior desafio enfrentado pela sociedade moderna e a economia de água, em processos produtivos, requer especial atenção devido ao valor agregado que tem sido atribuído a este bem ${ }^{[2-5]}$. Neste contexto, se insere o setor têxtil brasileiro com um grande parque industrial instalado, contando com cerca de 5000 indústrias ${ }^{[6]}$. Os efluentes líquidos dessas indústrias são potencialmente poluidores, pela presença de diversos compostos químicos utilizados na preparação e beneficiamento de tecidos. Estima-se que $20 \%$ dos corantes usados no processo de tingimento podem ser descartados em efluentes, devido a perdas ocorridas durante o processo de fixação $\mathrm{o}^{[7]}$.

No tratamento de efluentes industriais são aplicadas técnicas fundamentadas em processos de coagulação, se- guidas de separação por flotação ou sedimentação, necessárias para remover material particulado. Em muitos casos as remoções de cores e de compostos orgânicos dissolvidos envolvem processos biológicos por sistemas de lodos $\operatorname{ativados}^{[8]}$. A maioria desses processos está associada à geração de grandes quantidades de resíduo sólido, denominado de lodo. Quanto mais eficiente for o sistema de retenção de particulados, maior será a quantidade de lodo gerado no final do processo. A separação do lodo nos sistemas de tratamento de efluentes é uma prática necessária e complexa, tornando crítico o acúmulo desse material, tendo em vista que enormes volumes de lodo úmido terão que ser cuidadosamente armazenados. Esse fator é ainda mais agravante considerando-se a redução da disponibilidade de áreas próprias para a disposição de rejeitos industriais. A disposição final do lodo industrial exige processamento adequado e acondicionamento em condições especiais tornando o processo oneroso ${ }^{[9,10]}$.

O lodo pode ter diferentes disposições finais, dentre elas a incineração, a reciclagem via utilização agronômica 
na vermicompostagem ${ }^{[11]}$ e a incorporação em artefatos de cimento $^{[12]}$. No contexto do aproveitamento de rejeitos industriais, uma alternativa atraente é a preparação de compósitos estruturais. Os compósitos são classificados como materiais heterogêneos formados basicamente pela matriz que é a fase contínua e de maior fração volumétrica na mistura e a fase dispersa que é formada principalmente por componentes estruturais. Compósitos poliméricos são usados em larga escala tanto em utensílios domésticos, como nos materiais ligados às chamadas tecnologias de ponta ${ }^{[13,14]}$. Entre os materiais mais conhecidos na preparação de compósitos estruturais está a fibra de vidro. É um componente de reforço de baixo custo e de elevada resistência à tração. Como desvantagens, a fibra de vidro é abrasiva e quimicamente estável, dificultando a sua decomposição no meio ambiente ${ }^{[15,16]}$. Devido ao largo uso de compósitos poliméricos e a variedade de materiais empregados, pesquisas avançam no sentido da substituição de componentes tradicionais por outros alternativos de menor custo, como matrizes biodegradáveis reforçados com fibras vegetais ${ }^{[17-27]}$. O uso de resíduos industriais também é uma importante alternativa para minimizar impactos ambientais negativos. Um exemplo é o emprego de resíduos de ardósia como carga em matriz de polipropileno que resulta em compósitos com excelentes propriedades mecânicas ${ }^{[28]}$.

No presente artigo, está sendo destacada a utilização de lodo industrial têxtil na preparação de compósitos com matriz de poliéster insaturado. Outro tipo de material empregado neste trabalho foi a fibra de crisotila pura. Crisotila é utilizada na preparação de cimento-amianto (Fibrocimento). Artefatos de fibrocimento como telhas e caixas d'água foram largamente difundidos no passado recente. Atualmente, o cimento-amianto está em desuso, e por este motivo, grandes volumes de rejeitos estão sendo descartados. O uso da crisotila pura nos compósitos ao invés de resíduo de cimentoamianto tem como objetivo a montagem de sistemas mais simplificados, tendo em vista que o cimento-amianto é composto de areia, cimento e aditivos.

Entre os diferentes tipos de polímeros utilizados como matrizes de compósitos, os termofixos são responsáveis pela maior demanda. O baixo custo da matéria-prima e do processamento dos termofixos justificam o destaque em relação às matrizes termoplásticas ${ }^{[29]}$. Entre as principais matrizes termofixas está a resina de poliéster, em especial, a ortoftálica. Devido a estes fatores e ainda considerando as excelentes propriedades deste polímero, quando associado a fibras estruturais $\mathrm{s}^{[30]}$, neste trabalho foi empregada uma resina de poliéster ortoftálica como matriz polimérica.

\section{Experimental}

Os compósitos foram preparados com diferentes percentuais de fibras de vidro (FV), lodo (L) e fibras de crisotila (FC). Como matriz polimérica foi usada resina poliéster (P) ortoftálica UC 2120-AC-Plus, adquirida no comércio varejista e usada como suprida. Como componentes de reforço foram empregadas fibras de crisotila (5R) e fibras de vidro tipo $\mathrm{E}$ (picada 3,0-6,0 $\mathrm{mm}$ ). O lodo utilizado foi coletado em sistemas de tratamento de efluentes têxteis de indústrias do município de Blumenau-SC, após processos de coagulação e separação. Os efluentes são compostos por um grande número de reagentes usados no beneficiamento das fibras têxteis: são corantes Reativos (remazol e procion), pequenas quantidades corantes dispersos, a cuba e pigmentos, goma a base de carboxi metil amido e álcool polivinílico, silicatos de sódio, estabilizadores orgânicos, tensoativos diversos (umectantes, detergentes, emulgadores, sequestrantes e dispersantes), ácido fórmico e oxálico, amaciantes, cloreto e sulfato de sódio. Portanto, na composição do lodo têxtil encontra-se parte dos produtos do beneficiamento, além de sulfato de alumínio $\left(\mathrm{Al}_{2}\left(\mathrm{SO}_{4}\right)_{3}\right)$ e polieletrólitos catiônico, que são usados como agentes floculantes durante o processo de tratamento do efluente. O lodo utilizado neste estudo apresentou umidade de $80 \%$, cor cinza após a desidratação, odor repulsivo quando úmido e significativamente ameno após desidratação. Para atender um acordo firmado entre as empresas envolvidas no projeto e o grupo de pesquisa, a completa caracterização do lodo só será divulgada em trabalhos posteriores. Antes de ser usado nas formulações o lodo foi desidratado em estufa à vácuo por 12 horas à $80^{\circ} \mathrm{C}$. O material desidratado foi triturado em moinho de bolas, até atingir perfil granulométrico entre 0,090 e $0,150 \mathrm{~mm}$.

Os compósitos foram preparados por mistura mecânica (Misturador Mecânico Fisatom, modelo 713D) por 1 hora a $25{ }^{\circ} \mathrm{C}$, em ambiente com umidade relativa em torno de $50 \%$. O material homogêneo e catalisado com peróxido de metil etil cetona foi despejado em moldeiras para ensaios de resistência ao impacto (ASTM D 256-78), onde permaneceu por 24 horas. As condições de catálise foram previamente testadas. Para tanto, foram utilizadas diferentes composições submetidas a variações na temperatura e na quantidade de catalisador. Todas as composições apresentaram boa reticulação utilizando $1 \%$ de catalisador em relação à massa de resina poliéster. Quanto à temperatura, todas as composições sofreram reticulação a $25{ }^{\circ} \mathrm{C}$. As composições do tipo $\mathrm{P} / \mathrm{L}$ sem fibras sofreram cura após 3 horas enquanto que, o tempo para composições do tipo P/L/FC foi de 24 horas. Portanto, o tempo definido como padrão para a desmoldagem dos corpos-de-prova (CPs), para todas as composições testadas, foi de 24 horas. As composições usadas na caracterização por análises térmicas, densidade e resistência ao impacto estão relacionadas na Tabela 1 .

A condutividade térmica $(\mathrm{K})$ foi determinada conforme norma ABNT NBR 12094. As análises foram realizadas com CPs medindo $128 \mathrm{~mm} \times 100 \mathrm{~mm} \times 3 \mathrm{~mm}$. O material testado foi submetido a uma fonte térmica de $40 \mathrm{~W}$ por 180 minutos, com tomadas de temperatura a cada 5 minutos. A condutividade térmica foi calculada de acordo com a Equação 1, to- 
Tabela 1. Composições dos compósitos analisados.

\begin{tabular}{lcccrccc}
\hline \multicolumn{1}{c}{ Componentes } & \multicolumn{7}{c}{ Composições (\%) } \\
\hline Resina poliéster & 70 & 70 & 70 & 70 & 70 & 70 & 70 \\
Lodo industrial & 30 & - & - & 5 & 25 & 5 & 25 \\
Fibra de crisotila & - & 30 & - & 25 & 5 & - & - \\
Fibra de vidro & - & - & 30 & - & - & 25 & 5 \\
\hline
\end{tabular}

mando como base de cálculo para a média de três ensaios para cada composição.

$$
\frac{Q}{\Delta t}=K A \frac{\Delta T}{L}
$$

onde: $\frac{Q}{\Delta t}=$ calor transferido em função do tempo $(\mathrm{J} / \mathrm{s})$; $\mathrm{K}=$ condutividade térmica $(\mathrm{W} / \mathrm{mK}) ; \mathrm{A}=$ área do $\mathrm{CP}\left(\mathrm{m}^{2}\right)$; $\Delta \mathrm{T}=$ Variação de temperatura $(\mathrm{K}) ; \mathrm{L}=$ espessura do $\mathrm{CP}(\mathrm{m})$.

A densidade dos compósitos foi determinada conforme a norma ASTM D 792-66, em balança analítica Sartorios modelo BP 210 S, com 5 CPs para cada composição. As determinações da resistência ao impacto Izod foram executadas conforme norma ASTM D 256-78, a $25^{\circ} \mathrm{C}$ num aparelho EMIC modelo AIC, com $10 \mathrm{CPs}$ para cada composição. O comportamento termogravimétrico foi investigado em equipamento Shimadzu Mod. TG-50. As amostras com aproximadamente $10 \mathrm{mg}$ acondicionadas em cápsulas de platina, foram submetidas a corridas entre 25 e $700{ }^{\circ} \mathrm{C}$, com taxa de aquecimento de $10^{\circ} \mathrm{C} / \mathrm{min}$, sob atmosfera de $\mathrm{N}_{2}(50 \mathrm{~mL} / \mathrm{min})$.

\section{Resultados e Discussão}

Os resultados obtidos dos ensaios de densidade estão apresentados na Tabela 2, onde se pode observar que a adição de lodo diminuiu a densidade de todos os compósitos. Isto se deve ao fato de ser o lodo menos denso do que as fibras, conforme demonstrado nas determinações da densidade da composição P/L 70/30, cujo resultado foi $1,268 \mathrm{~g} / \mathrm{cm}^{3}$. A incorporação de $5 \%$ de lodo no compósito com fibra de crisotila não afetou significativamente a densidade do compósito $(0,5 \%)$ enquanto que, com $25 \%$ de lodo a densidade diminuiu em $5,1 \%$. Para compósitos com fibra de vidro o efeito foi mais pronunciado, chegando a reduzir a densidade dos compósitos em até 13,6\%.

Um dos efeitos mais importantes na preparação de materiais estruturais é a redução da densidade. Materiais leves promovem a economia de energia que está associada diretamente com a diminuição da massa do produto acabado. Artefatos de compósitos desenvolvidos pela indústria aeronáutica e automobilística são exemplos típicos do uso de materiais

Tabela 2. Variação da densidade (d) em função do percentual de lodo.

\begin{tabular}{lc}
\hline Composição & Densidade $\left(\mathbf{g} / \mathbf{c m}^{\mathbf{3}}\right)$ \\
\hline $\mathrm{P} / \mathrm{FC} \mathrm{70/30}$ & 1,395 \\
$\mathrm{P} / \mathrm{L} / \mathrm{FC} \mathrm{70/5/25}$ & 1,388 \\
$\mathrm{P} / \mathrm{L} / \mathrm{FC} \mathrm{70/25/5}$ & 1,324 \\
$\mathrm{P} / \mathrm{FV} \mathrm{70/30}$ & 1,416 \\
$\mathrm{P} / \mathrm{L} / \mathrm{FV} \mathrm{70/5/25}$ & 1,328 \\
$\mathrm{P} / \mathrm{L} / \mathrm{FV} \mathrm{70/25/5}$ & 1,224 \\
\hline
\end{tabular}

de baixa densidade ${ }^{[32,33]}$. Os valores relacionados na Tabela 2 são importantes neste aspecto, pois demonstram ganhos na redução da densidade dos compósitos na medida em que o percentual de lodo aumenta.

Em relação à resistência ao impacto para as composições contendo lodo, verificou-se que ocorreram perdas, ou seja, na medida em que o percentual de lodo aumentou a resistência ao impacto diminuiu. Esta tendência pode ser visualizada nos dados da Tabela 3 que relacionam o aumento de 5\% para $25 \%$ de lodo nas composições com fibras de vidro e crisotila. Portanto, o lodo não se comporta como componente de reforço, caso contrário os valores da resistência ao impacto dos compósitos com 25\% de lodo seriam maiores. Estas indicações são confirmadas pelo resultado para a composição P/L 70/30 com o valor 7,646 J.m-1 , que é mais baixo do que qualquer outro valor apresentado na Tabela 3. No entanto, este comportamento já era esperado devido ao fato de ser o lodo um material pulverulento com baixos teores de fibras. Os resultados de resistência ao impacto obtidos por Ferro ${ }^{[32]}$, também mostram que materiais pulverulentos como cinzas de casca de arroz e talco não se comportam como componente de reforço para matrizes de naílon6 e náilon66. Analisando detalhadamente os resultados da Tabela 3 constata-se que, nos compósitos com fibra de crisotila a perda mecânica máxima foi de $66,9 \%$, enquanto que com FV foi de $57 \%$, indicando que a fibra de vidro promove maior efeito estrutural do que a fibra de crisotila.

A condutividade térmica $(\mathrm{K})$ dos compósitos se caracterizou pelo efeito que cada tipo de componente estrutural (lodo, fibra de crisotila e fibra de vidro) produz sobre a capacidade de isolamento térmico. Este efeito pode ser acompanhado na Tabela 4 que relaciona a variação da condutividade térmica dos laminados com cada componente estrutural.

O compósito $\mathrm{P} / \mathrm{L}$ com o menor valor de $\mathrm{K}$ é o material com maior capacidade de isolamento térmico. Para evidenciar esta propriedade nos compósitos a base de lodo, foram montados experimentos com compósitos do tipo $\mathrm{P} / \mathrm{L} / \mathrm{FC}$, com variações na proporção de lodo e fibras de crisotila. Os resultados apresentados na Figura 1 confirmam que o aumento do percentual de lodo aumenta a eficiência do material como isolador térmico. Para compósitos do tipo P/L/FC, com $25 \%$ de lodo a temperatura de estabilização foi de $49^{\circ} \mathrm{C}$, enquanto que, com $5 \%$ de lodo a temperatura foi de $53{ }^{\circ} \mathrm{C}$.

Tabela 3. Valores médios de resistência ao impacto.

\begin{tabular}{lc}
\hline Composição & Resistência ao impacto ${ }^{\text {DP }}\left(\right.$ J.m $\left.^{-1}\right)$ \\
\hline P/FC 70/30 & $32,46^{0,10}$ \\
P/L/FC 70/5/25 & $29,11^{0,45}$ \\
P/L/FC 70/25/5 & $10,74^{0,32}$ \\
P/FV 70/30 & $151,50^{0,20}$ \\
P/L/FV 70/5/25 & $99,50^{0,28}$ \\
P/L/FV 70/25/5 & $65,30^{0,30}$ \\
\hline
\end{tabular}

$\mathrm{DP}=$ desvio padrão.

Tabela 4. Valores médios da condutividade térmica.

\begin{tabular}{cccc}
\hline Composição (\%) & P/L 70/30 & P/FC 70/30 & P/FV 70/30 \\
\hline $\mathrm{K}\left(\mathrm{Wm}^{-1} \mathrm{~K}^{-1}\right)$ & 0,00161 & 0,00172 & 0,00198 \\
\hline
\end{tabular}


A influência da incorporação de lodo também foi investigada por análise termogravimétrica em atmosfera inerte $\left(\mathrm{N}_{2}\right)$ com taxa de aquecimento de $10^{\circ} \mathrm{C} \mathrm{min}^{-1}$. Foram avaliadas amostras com dois e três componentes: P/FV, P/L/FV, P/FC e P/L/FC. As análises dos resultados indicados pelas curvas termogravimétricas nas Figuras 2, 3, 4 e 5 permitem concluir que a incorporação de lodo não aumentou a resistência térmica das composições, visto que as temperaturas máximas de decomposição são muito próximas tanto para os compósitos sem lodo (Figura 2 e 3),

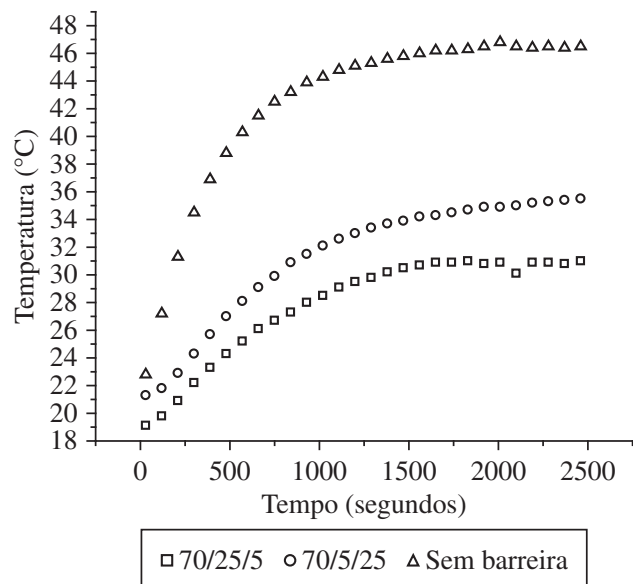

Figura 1. Variação da temperatura em função do tempo para compósitos do tipo $\mathrm{P} / \mathrm{L} / \mathrm{FC}$.

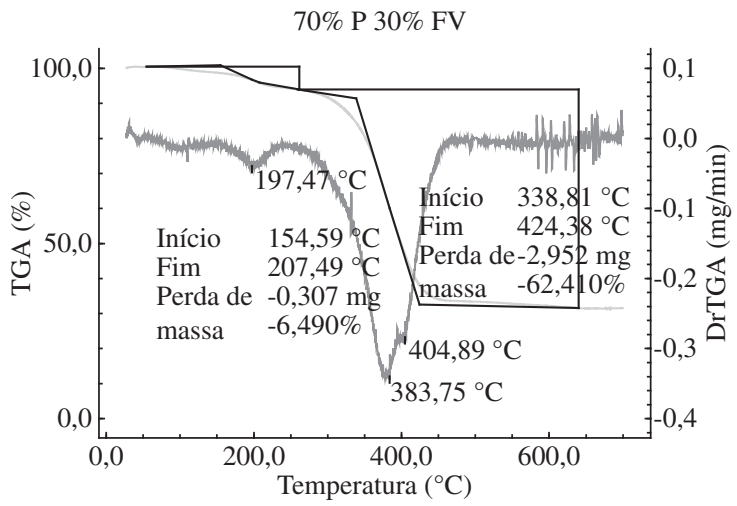

Figura 2. Curvas termogravimétricas para P/FV 70/30.

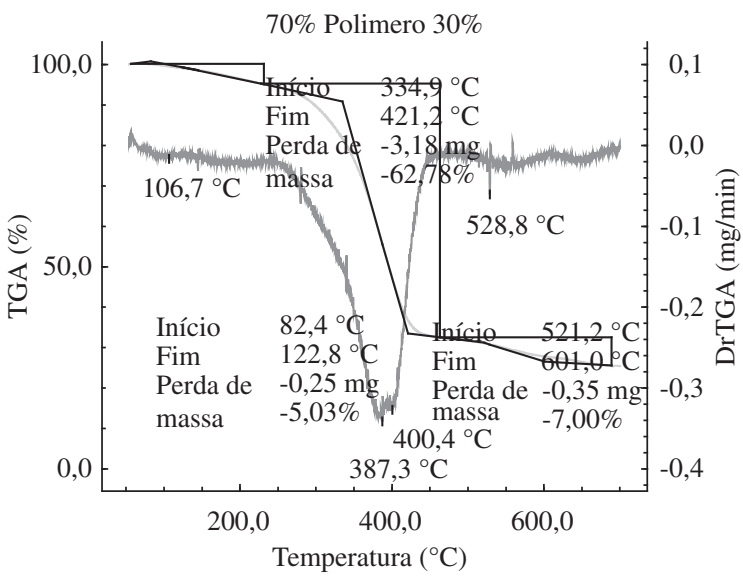

Figura 3. Curvas termogravimétricas para P/FC 70/30.

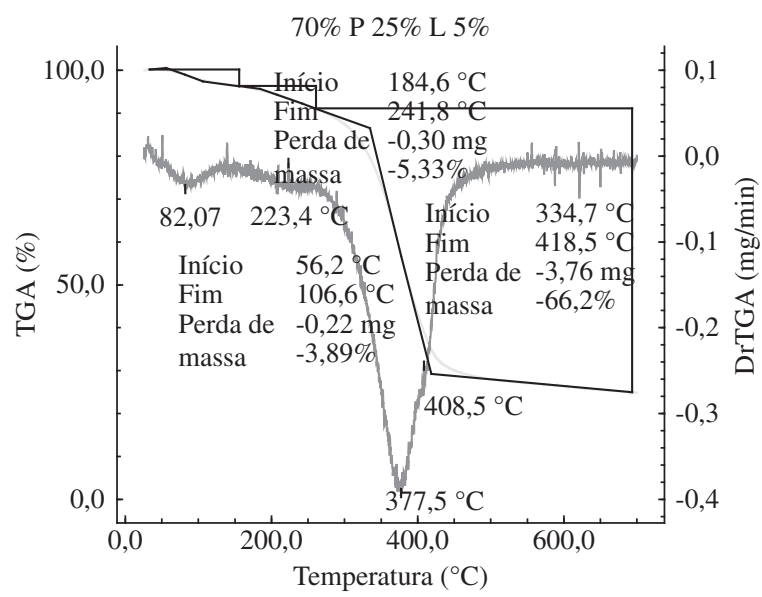

Figura 4. Curvas termogravimétricas para P/L/FV 70/25/5.

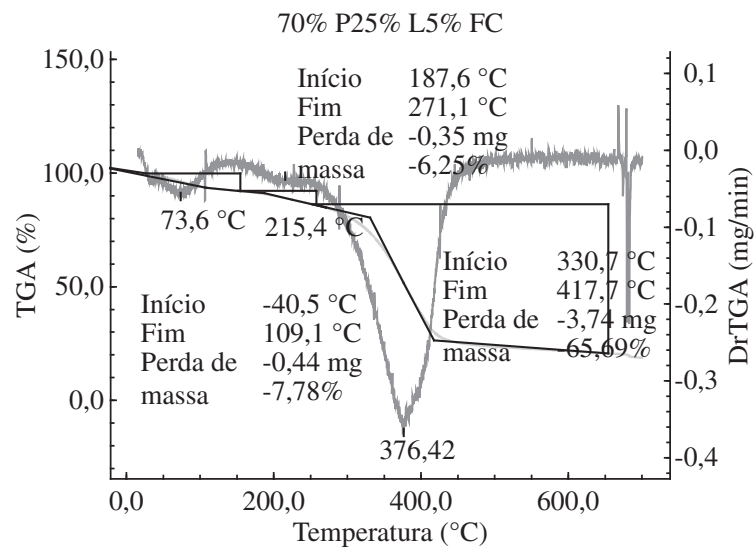

Figura 5. Curvas termogravimétricas para P/L/FC 70/25/5.

como para os compósitos com lodo (Figura 4 e 5). As variações de perda de massa provocadas pela adição do lodo foram de apenas 3,8\% para $\mathrm{P} / \mathrm{L} / \mathrm{FV}$ e $2,9 \%$ para $\mathrm{P} / \mathrm{L} / \mathrm{FC}$ indicando que a estabilidade térmica não foi modificada.

Além destes aspectos, vale salientar que os comportamentos térmicos, representados pelos formatos das curvas TGA, são idênticos ao da resina pura indicando que as interações entre a matriz polimérica, fibras e lodo não são de alta energia, caso contrário seria possível observar maiores variações nas temperaturas de decomposição e variações do número de patamares das perdas de massa. Portanto, a presença dos comcomponentes de reforço e lodo garantiram a integridade térmica da matriz polimérica e provavelmente não provocou fortes interações químicas. A associação dos resultados discutidos neste artigo é uma contribuição importante para avaliar o potencial de aplicabilidade destes compósitos, principalmente associando a estabilidade térmica, a redução da densidade e a baixa condutividade térmica dos compósitos com maior percentual de lodo. A preparação de laminados leves para isolamento térmico de máquinas e fornos poderá ser uma alternativa viável. Outra possibilidade é a construção de acessórios para equipamentos usados em estações de tratamento de efluentes domésticos e industriais como: flutuadores para bombas de aeração e barreiras de contenção para líquidos. 


\section{Conclusão}

A preparação por mistura mecânica de compósitos à base de resina poliéster, fibras de vidro, fibras de crisotila e lodo industrial, resultou em laminados termicamente estáveis. Também é possível assegurar que o lodo promove redução da densidade nos compósitos, independentemente do tipo de material fibroso utilizado, ampliando as possibilidades de aplicação destes materiais. A caracterização térmica dos compósitos indicou que o percentual de lodo incorporado é proporcional à capacidade de isolamento térmico dos materiais, sem afetar negativamente a estabilidade térmica da matriz poliéster. Com relação às propriedades mecânicas, de forma geral, a incorporação de lodo provocou perdas na resistência ao impacto dos compósitos, indicando que este componente não se comporta como estrutura de reforço. No entanto, as perdas mecânicas foram menos acentuadas nos compósitos de crisotila com 5\% de lodo.

\section{Agradecimentos}

Os autores agradecem a colaboração do POLIMAT-UFSC na execução das análises térmicas, ao Professor Elcio Schuhmacher - FURB na orientação das análises mecânicas e a Pró-Reitoria de Pesquisa, Pós-Graduação e Extensão FURB pelo apoio financeiro e técnico.

\section{Referências Bibliográficas}

1. Bechtold, T.; Burtscher, E. \& Turcanu, E. - A. J. Chem. Tech. and Bio., 76, p.303 (2001).

2. Barcellos I. O.; Andreaus, J.; Battist, A. \& Borges, J. K. - Polím.: Ciência e Tecnol., 18, p.215 (2008).

3. Lucas, M.; Jeremias, P. F. P. T.; Andreaus, J.; Barcellos, I. O. \& Zamora, P. P. - Quím. Nova, 31, p.1362 (2008).

4. Chiarello, L. M.; Barcellos, I.O.; Faria, R. \& Blosfeld, A. M. - Quím. Têxtil, 91, p.54 (2008).

5. Mo, J. H.; Lee, Y. H.; Kim, J.; Jeong, J. Y. \& Jegal, J. Dyes and Pigm., 76, p.429 (2008).

6. Toledo, R. A. S. - Quím. Têxtil, 74, p.8 (2004).

7. Kunz, A.; Peralta-Zamora, P.; Moraes, S. G. \& Duran, N. - Quím. Nova, 78, p.25 (2002).

8. Balan, D. S. L. - Química Têxtil, 54, p.26 (2000).

9. Zanoni, M. V. B. \& Carneiro, P. A. - Ciên. H., 123, p.62 (2001).

10. Veras, L. \& Povinelli, J. - Eng. San. Amb., 9, p.218 (2004).

11. Bidone, F. R. A. - "A Vermicompostagem dos Resíduos Sólidos de Curtume, Brutos e Previamente Lixiviados, utilizando Composto de Lixo Orgânico Urbano como Substrato", Tese de Doutorado, Universidade de São Paulo, Brasil (1995).

12. Juarez, C.; Durán, A.; Valdez, P. \& Fajardo, G. - Build. Env., 42, p.1151 (2006).

13. Rezende, M. - Polím. Ciência e Tecnol.., 17, p.E4 (2007).
14. Cândido, M. G. \& Almeida, S. F. - Polím. Ciência e Tecnol., 10, p.31 (2000).

15. Neto, F. L. \& Pardini, L. C. - "Compósitos Estruturais Ciência e Tecnologia”, Ed. Edgar Blücher Ltda, São Paulo, (2006).

16. Matheus, M. A. - "Fiberglass - Aprenda Fibra de Vidro", Ed. Legus Summa Ltda, Ribeirão Preto, (2002).

17. Alvarez, V. A.; Terenzi, A.; Kenny, J. M. \& Vazquez, A. Polym. Eng. and Science, 44, p.1907 (2004).

18. Lai, M. D.; Li, J.; Yang, J; Liu, J. J.; Tong, X. \& Cheng, H. M. - Polym. Internat., 53, p.1479 (2004).

19. Shibata, S. \& Cao, Y.; Fukumoto, I. - Polym. Testing, 24, p.1005 (2005).

20. Bonelli, C. M. C.; Elzubair, A.; Suarez, J. C. M. \& Mano, E. B. - Polím.: Ciência e Tecnol., 15, p.256 (2005).

21. Carvalho, L. H. \& Cavalcanti, W. S - Polím.: Ciência e Tecnol., 16, p.33 (2006).

22. Takagi, H. \& Ichihara,Y. - JSME Intern. Journ. Series A-Solid Mech. and Mat. Eng., 47, p.551 (2004).

23. Mintova, S.; Valtchev, V.; Schoeman, B. \& Sterte, J. - J. Porous Mat., 3, p.143 (1996).

24. Balzer, P. L.; Vicente, L.; Briesemeister, R.; Becker, D.; Soldi, V.; Rodolfo, A. J. \& Feltran, M. B. - Polím.: Ciência e Tecnol., 17, p.1 (2007).

25. De Paoli, M. A.; Mano, B. \& Spinacé, M. A. - Compósitos de polipropileno com fibras de curauá processados em extrusora mono ou dupla- rosca - in: Anais da 29a Reunião Anual da Sociedade Brasileira de Química SBQ, Águas de Lindóia - SP, mai (2006).

26. Guimarães, J. L.; Satyanarayana, K. G.; Wypych, F. \& Ramos, L. P. - Thermoforming of starch-fiber composite laminates. Braz. Pedido PI (2007), p.6 CODEN: BPXXDX BR 2006002428.

27. Cinelli, P.; Chiellini, E. \& Imam, S. H. - Journ. of Appl. Polym. Sci., 109, p.1684 (2008).

28. Carvalho, G. M. X.; Mansur, H. S.; Vasconcelos, W. L. \& Oréfice, R. L. - Polím.: Ciência e Tecnol., 17, p.98 (2007).

29. Neto, F. L. \& Pardini, L. C. - "Compósitos Estruturais Ciência e Tecnologia", Ed. Edgar Blücher Ltda, São Paulo, (2006).

30. Silaex Química Ltda, Conteúdo Técnico, Disponível em: http://www.silaex.com.br/poliéster.htm.

31. Crespim, H.; Azevedo , M. F. P.; David , L. H.; Cassu, S. N. \& Lourenço, V. L. - Polím. Ciência e Tecnol., 17, p.228 (2007).

32. Ferro, W. P.; Silva, L. G. A. \& Wiebeck, H. - Polím. Ciência e Tecnol., 17, p.240 (2007).

33. Mazur, R. L.; Botelho, E. C.; Costa, M. L. \& Rezende, M. C. - Polím. Ciência e Tec. 18, p.237 (2008).

Enviado: 03/06/08

Reenviado: 10/03/09

Aceito: $17 / 03 / 09$ 